APPLIED PHYSICS LETTERS 86, 134101 (2005)

\title{
Miniaturized single-crystal silicon cantilevers for scanning force microscopy
}

\author{
J. L. Yang ${ }^{\text {a) }}$ \\ Laboratory, 8803 Rüschlikon, Switzerland \\ M. Despont ${ }^{\text {b) }}$ and U. Drechsler \\ IBM Research, Zurich Research Laboratory, 8803 Rüschlikon, Switzerland
}

Institute of Physics, University of Basel, 4056 Basel, Switzerland and IBM Research, Zurich Research

B. W. Hoogenboom

Institute of Physics, University of Basel, 4056 Basel, Switzerland and M. E. Müller Institute, Biozentrum, University of Basel, 4056 Basel, Switzerland

P. L. T. M. Frederix

M. E. Milller Institute, Biozentrum, University of Basel, 4056 Basel, Switzerland

S. Martin

Institute of Physics, University of Basel, 4056 Basel, Switzerland

A. Engel

M. E. Müller Institute, Biozentrum, University of Basel, 4056 Basel, Switzerland

P. Vettiger

IBM Research, Zurich Research Laboratory, 8803 Rüschlikon, Switzerland and Institute of Microtechnology, University of Neuchâtel, and CSEM, 2007 Neuchatel, Switzerland

\author{
H. J. Hug \\ Institute of Physics, University of Basel, 4056 Basel, Switzerland and Swiss Federal Laboratories for \\ Materials Testing and Research, EMPA, 8600 Dübendorf, Switzerland
}

(Received 17 November 2004; accepted 22 February 2005; published online 21 March 2005)

\begin{abstract}
We report on a method to reproducibly batch fabricate single-crystal silicon cantilevers with dimensions an order of magnitude smaller than conventional cantilevers, i.e., with lengths between 10 and $35 \mu \mathrm{m}$, a width of $4 \mu \mathrm{m}$, and a thickness of $0.2 \mu \mathrm{m}$. The cantilevers are attached to support chips specially tailored for use in a scanning force microscope. The resonance frequencies are $0.2-2 \mathrm{MHz}$, the spring constants about $0.05-1 \mathrm{~N} / \mathrm{m}$, and the quality factors $1.5-3.0 \times 10^{4}$. The resulting thermal force noise is $1-2 \times 10^{-16} \mathrm{~N} / \sqrt{\mathrm{Hz}}$ at room temperature in vacuum. These cantilevers allow an enhancement of the measurement speed of more than one order of magnitude, and $2-5 \times$ better force sensitivity. After annealing in ultrahigh vacuum and measuring at liquid helium temperature, the thermal force noise of these cantilevers is expected to be further reduced down to $10^{-18} \mathrm{~N} / \sqrt{\mathrm{Hz}}$. (C) 2005 American Institute of Physics. [DOI: 10.1063/1.1895482]
\end{abstract}

The scanning force microscope (SFM) has become an important tool for the characterization of a large variety of surfaces and for the measurement of forces of various origins. The central element of the SFM is a micromechanical cantilever, usually made of silicon or silicon nitride. The mechanical properties of the cantilever set limits for both the force sensitivity and the measurement speed of an SFM experiment.

First, the thermal energy in the cantilever leads to a white force noise given by

$$
\delta F_{\mathrm{th}}=\sqrt{\frac{2 k k_{B} T B}{\pi f_{\mathrm{res}} Q}},
$$

where $k$ is the spring constant of the cantilever, $f_{\text {res }}$ the resonance frequency, $Q$ the quality factor, $k_{B}$ Boltzmann's constant, $T$ the temperature, and $B$ the measurement

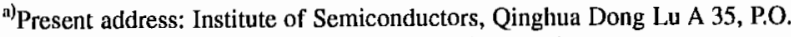
Box 912, Beijing 100083, People's Republic of China.

b) Author to whom correspondence should be addressed; electronic mail: dpt@zurich.ibm.com
}

bandwidth. ${ }^{1-3}$ Atomic-resolution imaging and force spectroscopy generally rely on detection of the cantilever frequency shift in the regime of attractive cantilever-sample forces, i.e., in noncontact mode. In this case, the minimum detectable force gradient is proportional to $\delta F_{\mathrm{th}}{ }^{2}{ }^{2}$ For intermittentcontact ("tapping") mode and contact ("constant force") mode, it is more difficult to give an expression for the force or the force-gradient sensitivity. However, $\delta F_{\text {th }}$ still imposes a lower limit for the minimum detectable force and for the minimum force that needs to be applied to the sample to obtain a reasonable signal-to-noise ratio. Therefore, to enhance the force sensitivity, one should reduce $\delta F_{\text {th }}$, which in terms of mechanical properties of the cantilever implies a low $k$, a high $f_{\text {res, }}$, and a high $Q$.

Second, $f_{\text {res }}$ sets an upper limit to the point or pixel frequency of the measurement, and thus to the measurement speed in general. To enhance the SFM imaging speed allowed by the cantilever, $f_{\text {res }}$ should therefore be increased. This should preferably be achieved without increasing $k$, as a larger $k$ implies a lower sensitivity [Eq. (1)]. The only way to combine a high $f_{\text {res }}$ with a low $k$ is by reducing the dimen- 


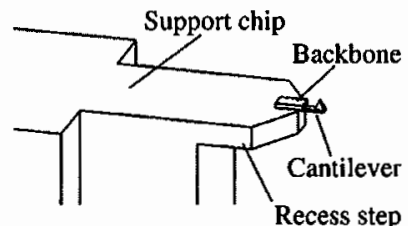

FIG. 1. Schematic of the geometry of the cantilever and its support chip, not to scale,

sions of the cantilever; however, support and surface losses make it increasingly difficult to obtain short and thin cantilevers with a high $Q$ in vacuum. ${ }^{3-5}$

In practice, it has been possible to reduce all cantilever dimensions by about an order of magnitude compared with those of conventional cantilevers, i.e., to $5-40 \mu \mathrm{m}$ in length, $4 \mu \mathrm{m}$ in width, and $0.1-0.2 \mu \mathrm{m}$ in thickness. For measurements in air and water, such cantilevers were manufactured out of silicon nitride. ${ }^{6}$ However, silicon-nitride cantilevers have the disadvantage of considerably lower $Q$ in vacuum, ${ }^{3}$ and usually exhibit a lower tip aspect ratio and sharpness than those made out of single-crystal silicon. A major challenge is to fabricate single-crystal silicon cantilevers $\mathrm{s}^{4,5,7,8}$ that combine reduced dimensions with a high $Q$ in vacuum and can be easily used in an SFM. The latter point imposes restrictions on the design of the anchoring/support chip to which the cantilever is attached. In particular, one should prevent the support chip from touching the sample before (the tip of) the cantilever makes contact. Therefore, at the point where the cantilever is attached, the chip should have a width comparable to the cantilever length. Furthermore, to detect the deflection of a small cantilever using optical methods, a small spot is required, and thus a large opening angle above the cantilever.

These requirements can be met with a design as that depicted in Fig. 1. The triangular shape of the support chip near the cantilever fixation allows sufficient clearance for the cantilever-sample approach. The optical opening angle is obtained by a recess step, with the maximum thickness pre-

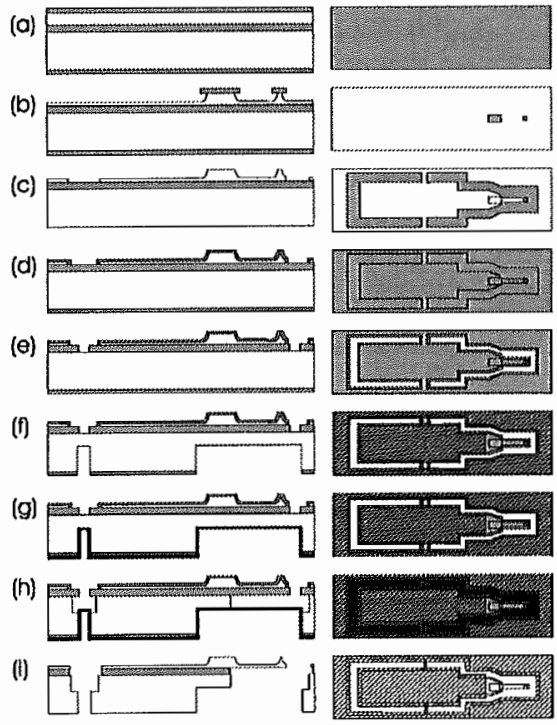

FIG. 2. Process flow chart showing the cross sections and the top views of the basic steps in the fabrication of the cantilever and its support chip. Light gray=silicon; dark gray = silicon oxide; black=aluminium .

Downloaded 18 Oct 2005 to 131.152 .32219 . Pedistribution subject to
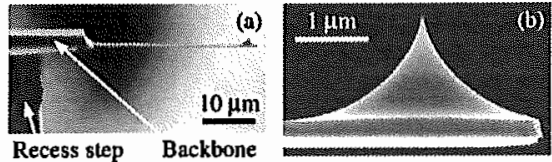

FlG. 3. (a) SEM image of a cantilever and part of the recess step, as fabricated with the process described in the text. (b) Close-up of the cantilever tip. The tip diameter is $<10 \mathrm{~nm}$.

defined by the cantilever length and the required opening angle (the full opening angle required for our detector is $\left.26^{\circ}\right)^{9}$. The chip is designed such that the recess step can be used as a shadow mask to evaporate a reflective coating only at the cantilever end. ${ }^{9,10}$ To reduce support loss, the cantilever widens, in a step-like fashion, into a rigid support structure (hereafter denoted as "backbone"), which in turn is attached to the support chip. Standing mechanical waves formed by the moving cantilever are thus reflected from the well-defined, backbone edge with minimum energy absorption. As an additional benefit, the backbone enables the cantilever length to be chosen with high precision. ${ }^{7}$ Finally, on top of the support chip, calibration marks are added to allow calibration of the piezo-motor used for the positioning of the deflection detector to the cantilever.

Figure 2 shows the process flow chart (cross section and top view) for the fabrication of the miniaturized cantilever and its support chip according to the design described above. The initial substrate is a 4 in. (100)-oriented silicon-oninsulator wafer with a $3.0-\mu$ m-thick Si membrane. On this membrane, a silicon oxide $\left(\mathrm{SiO}_{2}\right)$ layer is thermally grown in step (a). Next, this $\mathrm{SiO}_{2}$ layer is patterned with reactive ion etching (RIE), defining a mask for the tip and backbone fabrication. In step (b), isotropic RIE is used to form the backbone and a horn shape, which at the end of the process will result in a sharp tip owing to subsequent thermal oxidation. ${ }^{11}$ After wet etching the $\mathrm{SiO}_{2}$ mask, the cantilever is formed using RIE [step (c)], followed by thermal oxidation [step (d)] to protect the cantilever during the remaining process steps. Using RIE, in step (e) a front trench is created in the buried oxide around the cantilever and the support chip. From the backside of the wafer, the chip body is defined with deep RIE (DRIE), until the remaining Si has the desired thickness for the recess step [step (f)]. An aluminium (Al) layer, sputtered from the backside [ step $(\mathrm{g})]$, serves as an etch stop for

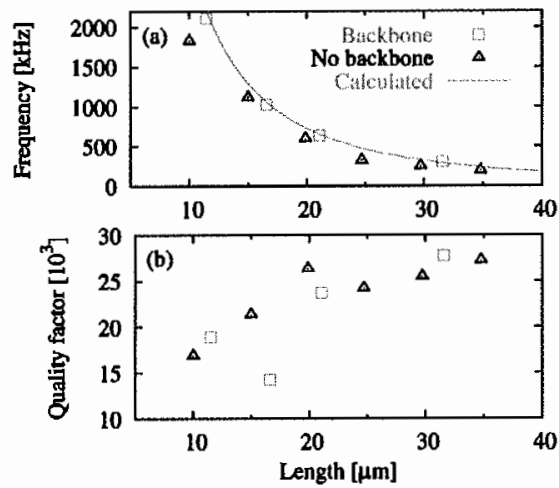

FIG. 4. (Color online). (a) $f_{\text {res }}$ and (b) $Q$ vs cantilever length for cantilevers with (squares) and without backbone (triangles). The curve in (a) is the theoretical resonance frequency based on the cantilever length, an average thickness of $0.21 \mu \mathrm{m}$, and the material properties of silicon (see Ref. 12). 
TABLE I. Typical results of the characterization of cantilevers (with backbone), at RT in vacuum.

\begin{tabular}{ccccc}
\hline \hline$\ell \times w \times t\left(\mu \mathrm{m}^{3}\right)$ & $f_{\text {rcs }}(\mathrm{kHz})$ & $k(\mathrm{~N} / \mathrm{m})$ & $Q$ & $\delta F_{\mathrm{ut}}{ }^{\mathrm{a}}(\mathrm{N} / \sqrt{\mathrm{Hz}})$ \\
\hline $11.5 \times 4.16 \times 0.20$ & 2112 & 1.0 & $\mathrm{t} .9 \times 10^{4}$ & $2.4 \times 10^{-16}$ \\
$21.1 \times 4.17 \times 0.20$ & 641 & 0.17 & $2.4 \times 10^{4}$ & $1.6 \times 10^{-16}$ \\
$31.6 \times 4.06 \times 0.24$ & 311 & 0.08 & $2.8 \times 10^{4}$ & $1.3 \times 10^{-16}$ \\
$253 \times 33.3 \times 2.45^{\mathrm{b}}$ & 41.6 & 1.4 & $1.7 \times 10^{5}$ & $5.2 \times 10^{-16}$ \\
\hline \hline
\end{tabular}

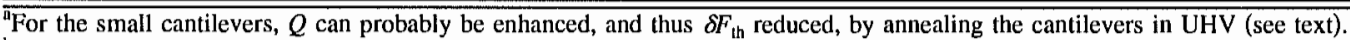

${ }^{b}$ Conventional cantilever (without backbone), Team Nanotec GmbH, Villingen-Schwenningen, Germany.

the front-side RIE that releases the cantilever in step (h). This etching is done in two phases: Anisotropic DRIE until the Al layer is reached, followed by isotropic RIE to underetch the Si below the cantilever. Because the cantilever is only $4 \mu \mathrm{m}$ wide, very little underetching is required to release it. Finally, the $\mathrm{Al}$ and the protective $\mathrm{SiO}_{2}$ layers of the cantilever are removed by two subsequent wet-etching steps [step (i)]. To study cantilever support loss, cantilevers without backbone have also been fabricated.

In conventional fabrication processes, the cantilever length is determined by the double-side alignment in step (f). In this case, a misalignment of $10-20 \mu \mathrm{m}$ can hardly be avoided. ${ }^{7}$ In our fabrication process, the cantilever length is determined by the front-side etching only, even for cantilevers without a backbone, resulting in an excellent control of the anchoring point (within 1-2 $\mu \mathrm{m}$ ). Our approach uses isotropic (under)etching to release the cantilever in step (h). The process is reproducible, and a fabrication yield $>70 \%$ has been obtained in our research environment. This demonstrates the robustness of the technique and its potential for commercial cantilever production.

Figure 3 shows scanning electron microscopy (SEM) images of a cantilever fabricated using the fabrication process. Cantilevers were characterized by taking resonance curves at room temperature (RT) in vacuum $\left(\$ 10^{-5} \mathrm{mbar}\right)$, using a detector built for measuring the deflection of micrometer-sized cantilevers. ${ }^{9}$ The cantilever dimensions were determined from SEM images, and $f_{\text {res }}$ and $Q$ from harmonic oscillator fits to the resonance curves. The value of $k$ was estimated from the cantilever dimensions and the modulus of elasticity of silicon. ${ }^{12}$ For each cantilever, $\delta F_{\text {th }}$ was calculated using Eq. (1). In Table I some typical results are given for cantilevers with backbone (those for cantilevers without backbone are similar) and compared with the data for a conventional cantilever with a similar $k$. In Fig. $4, f_{\text {res }}$ and $Q$ are shown for various cantilever lengths.

For small cantilevers with similar $k, f_{\text {res }}$ is 50 times larger than for the conventional cantilever. The $Q \mathrm{~s}$ are between 1.5 and $3.0 \times 10^{4}$; below $20 \mu \mathrm{m}$ cantilever length, $Q$ decreases with decreasing cantilever length. Furthermore, we have observed that $Q$ can drop by a factor of $2-3$ simply by exposing the cantilever to atmospheric pressure over some weeks, suggesting that $Q$ is mainly determined by surface loss, in agreement with previous studies of thin cantilevers. ${ }^{3-5}$ We do not observe any significant difference in $Q$ between cantilevers with and without backbone. However, the cantilever length is more precisely defined with backbone, as indicated by the better match between the calculated and the measured resonance frequencies in Fig. 4(a). $\delta F_{\text {th }}$ is a factor of 2-5 smaller than for the conventional cantilever, in the low $10^{-16} \mathrm{~N} / \sqrt{\mathrm{Hz}}$ range.
With their high $f_{\text {res }}$, the small cantilevers thus allow a substantial enhancement of the measurement speed. We also find a (more modest) reduction in the thermal force noise. Here, it is important to realize that for submicrometer-thick cantilevers, $Q$, whose value is mainly determined by surface loss, can be enhanced by as much as an order of magnitude by annealing the cantilevers in ultrahigh vacuum. ${ }^{3-5}$ As a consequence, we expect that after annealing the thermal noise will be reduced to the $10^{-18} \mathrm{~N} / \sqrt{\mathrm{Hz}}$ range at liquid helium temperature. So far, this could only be achieved with extremely soft cantilevers $\left(f_{\text {res }} \approx 2 \mathrm{kHz}, k<10^{-5} \mathrm{~N} / \mathrm{m}\right)$, which need to be operated perpendicular to the sample surface to avoid a snap in of the tip to the surface at small tip-sample distances. ${ }^{13}$

In conclusion, we have developed a method to reproducibly fabricate single-crystal silicon cantilevers with all dimensions about an order of magnitude smaller than those of conventional cantilevers. The support chip of the cantilever is designed such that the cantilevers can easily be used in SFM experiments. The small size of the cantilevers allows important improvements in measurement speed and sensitivity.

This work was supported by the Swiss Top Nano 21 program and the NCCR Nanoscale Science. The authors acknowledge Y. Pellmont for technical assistance and T. Ashworth for critically reading the manuscript.

'C. V. Heer, Statistical Mechanics, Kinetic Theory, and Stochastic Processes (Academic, New York, 1972).

${ }^{2}$ T. R. Albrecht, P. Grütter, D. Horne, and D. Rugar, J. Appl. Phys. 69, 668 (1991).

${ }^{3}$ K. Y. Yasumura, T. D. Stowe, E. M. Chow, T. Pfafman, T, W. Kenny, B. C. Stipe, and D. Rugar, J. Microelectromech. Syst. 11, 117 (2000).

${ }^{4}$ J. L. Yang, T. Ono, and M. Esashi, Appl. Phys. Lett. 77, 3860 (2000).

5J. L. Yang, T. Ono, and M. Esashi, J. Microelectromech. Syst. 11, 775 (2002).

${ }^{6}$ M. B. Viani, T. E. Schäffer, A. Chand, M. Rief, H. E. Gaub, and P. K. Hansma, J. Appl. Phys, 86, 2258 (1999).

${ }^{7}$ S. Hosaka, K. Etoh, A. Kikukawa, and H. Koyanagi, J. Vac. Sci. Technol. B 18, 94 (2000).

${ }^{8}$ H. Kawakatsu, D. Saya, A. Kato, K. Fukushima, H. Toshiyoshi, and H. Fujita, Rev. Sci. Instrum. 73, 1188 (2002).

${ }^{9}$ B. W. Hoogenboom, P. L. T. M. Frederix, J. L. Yang, S. Martin, Y. Pellmont, M. Steinacher, S. Zäch, E. Langenbach, H.-J. Heimbeck, A. Engel, and H. J. Hug (2004), Appl. Phys. Lett. 86, 074101 (2005).

${ }^{10}$ B. W. Hoogenboom, J. L. Yang, S. Martin, and H. J. Hug, European Patent No. file EP03,025,187 (4 November 2003).

${ }^{11} \mathrm{M}$. Despont, J. Brügger, U. Drechsler, U. Dlirig, W. Häberle, M. Lutwyche, H. Rothuizen, R. Stutz, R. Widmer, G. Binnig, H. Rohrer, and P. Vettiger, Sens. Actuators, A 80, 100 (2000).

${ }^{12}$ D. Sarid, Scanning Force Microscopy (Oxford University Press, Oxford, 1991).

${ }^{13}$ T. D. Stowe, K. Yasumura, T. W. Kenny, D. Botkin, K. Wago, and D. Rugar, Appl. Phys. Lett. 71, 288 (1997). 